(7) The triangle formulae for $\cos A$ and $\tan \frac{B-C}{2}$ are derivable in the same way. Also, by using the formulae corresponding to $\tan \frac{A}{2}=\sqrt{\frac{r r_{3}}{r_{2} r_{3}}}, \tan \frac{B-C}{2}$ can be shown equal to $\frac{r_{2}-r_{3}}{r_{1}+r} \tan \frac{A}{2}$.

As a final example, $\Sigma\left(\tan \frac{B}{2} \tan \frac{C}{2}\right)=\Sigma \sqrt{\frac{(s-a)(s-c)}{s(s-b)} \cdot \frac{(s-a)(s-b)}{s(s-c)}}=\Sigma\left(\frac{s-a}{s}\right)=1$ or

$$
\begin{gathered}
\Sigma\left(\tan \frac{B}{2} \tan \frac{C}{2}\right)=\Sigma \sqrt{\frac{r r_{2}}{r_{1} r_{3}} \cdot \frac{r r_{3}}{r_{1} r_{2}}}=\Sigma\left(\frac{r}{r_{1}}\right) \\
\therefore \quad \frac{1}{r}=\frac{1}{r_{1}}+\frac{1}{r_{2}}+\frac{1}{r_{3}} .
\end{gathered}
$$

A. G. BURGess.

The Distance of a given Point from a given Line.If $P\left(x^{\prime}, y^{\prime}\right)$ is the given point and $a x+b y+c=0$ the equation of the given line, the expression for the distance of $P$ from the line and the coordinates of the foot of the perpendicular from $P$ to the line can be obtained by projections as follows:-

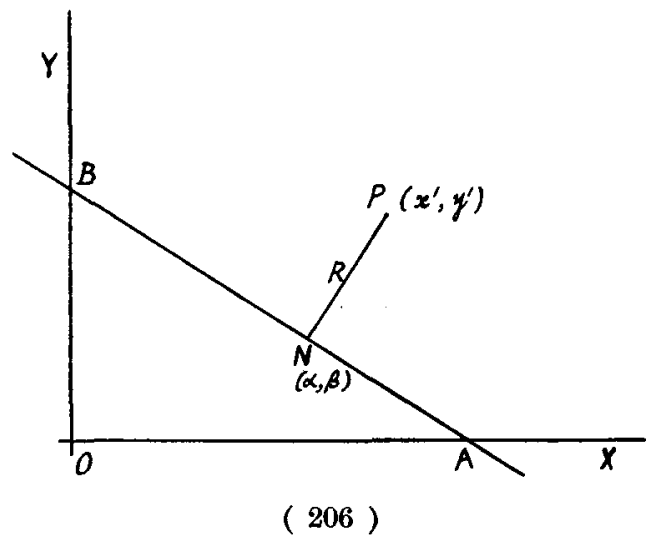


Let $N P=R$ and have projections $X$ and $Y$ on $O X$ and $O Y$ respectively. Then if $N P$ makes an angle $\theta$ with $O X$

$$
\begin{aligned}
X & =R \cos \theta \quad Y=R \sin \theta \\
\therefore \quad R & =X \cos \theta+Y \sin \theta .
\end{aligned}
$$

But

$$
\tan \theta=\text { gradient of } N Y^{\prime}=\frac{b}{a}
$$

$$
\therefore \quad \cos \theta=\frac{a}{d} \quad \text { and } \quad \sin \theta=\frac{b}{d} \text { where } d= \pm \sqrt{a^{2}+b^{2}} \text {. }
$$

Again, if $N$ is $(\alpha, \beta), X=x^{\prime}-\alpha$ and $Y=y^{\prime}-\beta$,

$$
\begin{aligned}
\therefore \quad R & =\frac{a\left(x^{\prime}-\alpha\right)+b\left(y^{\prime}-\beta\right)}{d} \\
& =\frac{a x^{\prime}+b y^{\prime}+c}{d}
\end{aligned}
$$

$$
\text { since } \quad \begin{array}{rlrl}
a \alpha+b \beta+c & =0 . \\
\text { Also } & x^{\prime}-\alpha & =R \cos \theta=\frac{a\left(a x^{\prime}+b y^{\prime}+c\right)}{d^{2}} \\
& \text { Hence } & \alpha & =x^{\prime}-\frac{a\left(a x^{\prime}+b y^{\prime}+c\right)}{a^{2}+b^{2}} \\
& \text { and } & \beta & =y^{\prime}-\frac{b\left(a x^{\prime}+b y^{\prime}+c\right)}{a^{2}+b^{2}}
\end{array}
$$

R. J. T. BELL.

\section{Note on the Determination of Centres of Curvature.} -For determining the Cartesian co-ordinates of the centre of curvature of a plane curve two methods are principally used in the text-books. One of these (see for example, Edwards, "Differential Calculus," p 266, § 339) having previously established the formula for the radius of curvature, derives the coordinates of the centre of curvature by using the circular functions of the angle " $\psi$ " which the tangent to the curve at the point considered makes with $O X$. Since, however, for the same tangent, and therefore the same " $\psi$," the curve may be either convex or concave towards $O X$ (and accordingly 